\title{
Vision-based relative state and inertia ratio estimation of un-cooperative
}

May 27-30, 2018, Toronto, On, Canada targets

\author{
Qian Feng, Quan Pan, Xiaolei Hou, Yong Liu \\ School of Automation \\ Northwestern Polytechnical University \\ Xi'an, Shannxi, 710072, China \\ qianfeng.nwpu@gmail.com, quanpan@nwpu.edu.cn
}

\author{
Zheng H. Zhu \\ Department of Mechanical Engineering \\ York University \\ Toronto, Ontario M3J 1P3 \\ gzhu@yorku.ca
}

\begin{abstract}
This paper develops an algorithm to estimate the relative pose, motion, and inertia ratio of an unknown target using stereoscopic vision. First, the positions and velocities of detected feature points on the spacecraft are estimated. Second, the angular velocity and attitude of the target are estimated by the least square method and q-method, respectively. Third, the position and velocity of the center of mass of the spacecraft are recovered. Finally, the inertia ratio is estimated based on the angular momentum conversation using the estimated angular velocity and attitude of the target. Numerical simulations are conducted to demonstrate the proposed algorithm.
\end{abstract}

Keywords-relative state; inertia ratio; un-cooperative spacecraft; stereoscopic vision; 3-D reconstruction; constrains.

\section{INTRODUCTION (HEADING 1)}

Estimating the dynamical parameters (pose and motion) and inertia property of an unknown target is one of the critical technologies during on-orbit autonomous close proximity operation missions [1], such as spacecraft rescuing, repairing, and capturing. These unknown targets are usually noncooperative without prior information about their structural and inertia parameters, nor artificial markers located known positions on the target. Therefore, the relative state and inertia properties are complete unknown. For a safe close proximity operation mission, such like approach to and capture the target, the relative state can provide important navigation information for the control strategy. Besides, the knowledge of inertia properties of the target is necessary to propagate its state and can be used in the motion prediction before capture and despun control design after capture.

Numerous vision-based estimation methods were proposed in the past [2-6] for non-cooperative targets. Terui et al. [7] proposed a 3D model matching method to estimate the relative state with the prior known structure of the target. Dehann et al. [8] adopted the stereo vision to measure the geometric center of the target as an approximation to its center of mass (CM). Du et al. [9] determined the pose of a large non-cooperative target based on two collaborative cameras by recognizing a rectangular feature on the target. Dong and Zhu $[10,11]$ estimated the pose and motion of a non-cooperative target with a monocular camera using some known feature point. Lichter and Dubowsky [12] presented a method to estimate the state using thousands of 3D points acquired from several cooperative 3D sensors, which leads to heavy computational efforts. Tweddle [13] proposed a Simultaneous Localization and Mapping (SLAM) solution to estimate the full state of a spinning non-cooperative target, with high computation complexity. Shay Segal [14] calculated the pose and motion with image position and velocity measurements of feature points on the target. By applying torque on the target produced from reaction wheels or a robotic arm, the inertia parameters are in-flight estimated $[15,16]$.

The purpose of this paper is to develop an innovative stereo vision-based algorithm to estimate the relative pose, motion, and inertia ratio of a non-cooperative target without prior knowledge about the positions of the feature points. The inertia ratio is estimated based on the conservation of angular momentum, assuming that the target is freely tumbling without any external torque on it.

The remainder of this paper is organized as follows. Section II provides the observation model. Section III gives the details of the proposed estimation algorithm. In Section IV, the newly proposed algorithm is validated by numerical simulation. Finally, Section V concludes the paper.

\section{OBSERVATION MODEL}

As depicted in Fig. 1, assume that two cameras mounted on the chaser are identical with parallel image planes and separated by a baseline $b$, the distance between the right camera's center of projection $\mathbf{C O P}_{R}$ and the left camera's center of projection $\mathbf{C O P}_{L}$. The target is in the field of view (FOV) of the both cameras. Two coordinate frames are used to describe the relative pose and motion of the unknown target: a) the target body frame $\mathcal{T}$ is a Cartesian frame with its origin at the CM of the target; b) the camera frame $\mathcal{C}$ is a Cartesian frame with its origin attached to $\mathbf{C O P}_{R}, \hat{\mathbf{x}}_{C}$ and $\hat{\mathbf{z}}_{C}$ axes parallel to the image plane and $\hat{\mathbf{y}}_{\mathcal{C}}$ axis pointing towards to the target. Here the frame $\mathcal{C}$ is assumed to be aligned with the inertial frame. The position and attitude of the target are defined by the vector $\boldsymbol{\rho}_{0}$ in the frame $\mathcal{C}$ and the direction cosine matrix $\mathbf{R}_{c}^{t}$ 
converting vectors from the frame $\mathcal{C}$ to the frame $\mathcal{T}$. The corresponding translational and rotational velocities are denoted as $\dot{\boldsymbol{\rho}}_{0}$ and $\boldsymbol{\omega}_{t c}^{c}$, respectively.

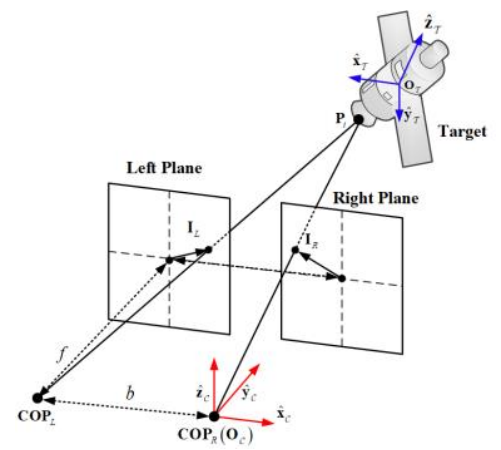

Fig. 1. Stereo vision measurement system.

Assuming that an arbitrary point $P_{i}$ on the target has the 3D coordinates $\boldsymbol{\rho}_{i}=\left[\begin{array}{lll}\rho_{i x} & \rho_{i y} & \rho_{i z}\end{array}\right]^{T}$ in the frame $\mathcal{C}$ and the $2 \mathrm{D}$ images coordinates $\mathbf{I}_{R}=\left[\begin{array}{ll}u_{i R} & v_{i R}\end{array}\right]^{T}$ and $\mathbf{I}_{L}=\left[\begin{array}{ll}u_{i L} & v_{i L}\end{array}\right]^{T}$ in the right and left image frames, respectively. According to the ideal pinhole camera model, the perspective projection transforms the feature point $P_{i}$ from the 3-D space onto the 2$\mathrm{D}$ image plan, such that

$$
\boldsymbol{\eta}_{i}=\left[\begin{array}{c}
u_{i R} \\
v_{i R} \\
u_{i L} \\
v_{i L}
\end{array}\right]=\left[\begin{array}{c}
f \frac{\rho_{i x}}{\rho_{i y}} \\
f \frac{\rho_{i z}}{\rho_{i y}} \\
f \frac{\rho_{i x}+b}{\rho_{i y}} \\
f \frac{\rho_{i z}}{\rho_{i y}}
\end{array}\right]
$$

where $f$ is the focal length.

Define the disparity as

$$
d_{i} \triangleq u_{i L}-u_{i R}
$$

The image velocity of the feature point is defined as the time derivative of its image coordinates,

$$
\left[\begin{array}{c}
\dot{u}_{i R} \\
\dot{v}_{i R} \\
\dot{u}_{i L} \\
\dot{v}_{i L}
\end{array}\right]=\frac{1}{\rho_{i y}}\left[\begin{array}{ccc}
f & -u_{i R} & 0 \\
0 & -v_{i R} & f \\
f & -u_{i L} & 0 \\
0 & -v_{i L} & f
\end{array}\right] \dot{\boldsymbol{\rho}}_{i}
$$

Considering the image noise, denoted by $\boldsymbol{\varepsilon}$, the images coordinates with noise could be modeled as

$$
\overline{\boldsymbol{\eta}}=\boldsymbol{\eta}+\boldsymbol{\varepsilon}
$$

where $\overline{\boldsymbol{\eta}}=\left[\bar{u}_{i R}, \bar{v}_{i R}, \bar{u}_{i L}, \bar{v}_{i L}\right]^{T}$ is the image measurement and $\boldsymbol{\varepsilon}_{i}$ is a zero-mean white noise with covariance matrix $\mathbf{R}_{i}$, i.e., $\boldsymbol{\varepsilon}_{i} \sim \mathcal{N}\left(0, \mathbf{R}_{i}\right)$.

\section{DESIGN AND IMPLEMENTATION OF STATE AND STRUCTURE ESTIMATION OF TARGET}

\section{A. Positions and velocities of feature points}

The positions of feature points are estimated by

$$
\overline{\boldsymbol{\rho}}_{i}=\left[\begin{array}{c}
\bar{\rho}_{i x} \\
\bar{\rho}_{i y} \\
\bar{\rho}_{i z}
\end{array}\right]=\left[\begin{array}{c}
\frac{b\left(\bar{u}_{i L}+\bar{u}_{i R}\right)}{2 \bar{d}_{i}}-\frac{b}{2} \\
\frac{b f}{\bar{d}_{i}} \\
\frac{b\left(\bar{v}_{i L}+\bar{v}_{i R}\right)}{2 \bar{d}_{i}}
\end{array}\right]
$$

The velocities of feature points are determined by

$$
\overline{\boldsymbol{\rho}}_{i}=C_{i}^{+} \boldsymbol{\xi}_{i}
$$

where the superscript "+" denotes the Moore-Penrose inversion of a matrix, and

$$
C_{i}=\frac{1}{\bar{\rho}_{i y}}\left[\begin{array}{ccc}
f & -\bar{u}_{i R} & 0 \\
0 & -\bar{v}_{i R} & f \\
f & -\bar{u}_{i L} & 0 \\
0 & -\bar{v}_{i L} & f
\end{array}\right], \quad \xi_{i}=\left[\begin{array}{c}
\overline{\dot{u}}_{i R} \\
\dot{\bar{v}}_{i R} \\
\dot{\vec{u}}_{i L} \\
\dot{\dot{v}}_{i L}
\end{array}\right] .
$$

\section{B. Pose and motion estimation of the target}

At time instant $t$, the relationship between $\boldsymbol{\rho}_{i}$ and $\mathbf{r}_{i}$ is expressed as:

$$
\boldsymbol{\rho}_{i}(t)=\boldsymbol{\rho}_{0}(t)+\mathbf{R}_{t}^{c}(t) \mathbf{r}_{i}
$$

where $\mathbf{R}_{t}^{c}=\left(\mathbf{R}_{c}^{t}\right)^{T}$ is the rotation matrix that transforms vectors from the frame $\mathcal{T}$ to the frame $\mathcal{C}$. Taking the time derivative of (7) yields

$$
\dot{\boldsymbol{\rho}}_{i}(t)=\dot{\boldsymbol{\rho}}_{0}(t)+\boldsymbol{\omega}_{t c}^{c}(t) \times\left[\mathbf{R}_{t}^{c}(t) \mathbf{r}_{i}\right]
$$

Defining $\delta \boldsymbol{\rho}_{i}(t)=\boldsymbol{\rho}_{i}(t)-\boldsymbol{\rho}_{1}(t)$ and $\delta \mathbf{r}_{i}=\mathbf{r}_{i}-\mathbf{r}_{1}$ yield

$$
\left\{\begin{array}{l}
\delta \boldsymbol{\rho}_{i}(t)=\mathbf{R}_{t}^{c}(t) \delta \mathbf{r}_{i} \\
\delta \dot{\boldsymbol{\rho}}_{i}(t)=\boldsymbol{\omega}_{t c}^{t}(t) \times\left[\mathbf{R}_{\mathrm{t}}^{c}(t) \delta \mathbf{r}_{i}\right], \quad 1 \leq i \leq N-1
\end{array}\right.
$$

Eliminating $\mathbf{R}_{t}^{c}(t) \delta \mathbf{r}_{i}^{t}$ in (9) leads to

$$
\left[\delta \boldsymbol{\rho}_{i}(t) \times\right] \boldsymbol{\omega}_{t c}^{c}(t)=-\delta \dot{\boldsymbol{\rho}}_{i}(t)
$$

where $[\mathbf{a} \times]$ is the cross-product matrix expressed as

$$
[\mathbf{a} \times]=\left[\begin{array}{ccc}
0 & -a_{3} & a_{2} \\
a_{3} & 0 & -a_{1} \\
-a_{2} & a_{1} & 0
\end{array}\right]
$$

The angular velocity of the target is estimated using LS method by

$$
\overline{\mathbf{\omega}}_{t c}^{c}(t)=\left[\mathbf{J}(t) \mathbf{J}(t)^{T}\right]^{-1} \mathbf{J}(t)^{T} \mathbf{b}(t)
$$

where $N$ is the number of feature points, and 


$$
\mathbf{J}(t)=\left[\begin{array}{c}
{\left[\delta \boldsymbol{\rho}_{1}(t) \times\right]} \\
\vdots \\
{\left[\delta \boldsymbol{\rho}_{N-1}(t) \times\right]}
\end{array}\right], \mathbf{b}(t)=\left[\begin{array}{c}
-\delta \dot{\boldsymbol{\rho}}_{1}(t) \\
\vdots \\
-\delta \dot{\boldsymbol{\rho}}_{N-1}(t)
\end{array}\right] .
$$

According to (9), the following relationship holds,

$$
\left\{\begin{array}{l}
\delta \boldsymbol{\rho}_{i}\left(t_{k}\right)=\mathbf{R}_{t}^{c}\left(t_{k}\right) \delta \mathbf{r}_{i} \\
\delta \boldsymbol{\rho}_{i}\left(t_{0}\right)=\mathbf{R}_{t}^{c}\left(t_{0}\right) \delta \mathbf{r}_{i}
\end{array}, \quad 1 \leq i \leq N-1\right.
$$

where $t_{k}=t_{0}+k \Delta t, \Delta t$ is the sample interval time, and $k$ is a positive integer.

Eliminating $\delta \mathbf{r}_{i}^{t}$ in (13) gives

$$
\delta \mathbf{\rho}_{i}\left(t_{k}\right)=\mathbf{R}_{t}^{c}\left(t_{k}\right)\left[\mathbf{R}_{t}^{c}\left(t_{0}\right)\right]^{T} \delta \mathbf{\rho}_{i}\left(t_{0}\right), 1 \leq i \leq N-1
$$

Define $\delta \mathbf{R}_{t}^{c}\left(t_{k, 0}\right)=\mathbf{R}_{t}^{c}\left(t_{k}\right)\left[\mathbf{R}_{t}^{c}\left(t_{0}\right)\right]^{T}$ as the attitude change from $t_{0}$ to $t_{k}$, then (14) is rewritten as

$$
\delta \boldsymbol{\rho}_{i}\left(t_{k}\right)=\delta \mathbf{R}_{t}^{c}\left(t_{k, 0}\right) \delta \boldsymbol{\rho}_{i}\left(t_{0}\right), 1 \leq i \leq N-1
$$

Equation (15) is solved by the q-method. Choose a set of nonnegative weights $\left\{a_{i}\right\}, i=1,2 \ldots, N-1$ and define

$$
\mathbf{B}=\sum_{i=1}^{N-1} a_{i} \delta \mathbf{\rho}_{i}\left(t_{k}\right)\left[\delta \boldsymbol{\rho}_{i}\left(t_{0}\right)\right]^{T}, \quad \mathbf{L}(\mathbf{B})=\left[\begin{array}{cc}
\mathbf{B}+\mathbf{B}^{\mathrm{T}}-(t r \mathbf{B}) \mathbf{I}_{3} & \mathbf{z} \\
\mathbf{z}^{\mathbf{T}} & \operatorname{tr} \mathbf{B}
\end{array}\right] .
$$

where $\mathbf{z}=\sum_{i=1}^{N-1} a_{i}\left[\delta \boldsymbol{\rho}_{i}\left(t_{k}\right) \times \delta \boldsymbol{\rho}_{i}\left(t_{0}\right)\right]$.

The optimal estimation $\delta \hat{\mathbf{q}}_{c t}\left(t_{k, 0}\right)$ is the eigenvector corresponding to the largest eigenvalue of $\mathbf{L}(\mathbf{B})$. Given the initial relative attitude, the relative attitude quaternion of the frame $\mathcal{C}$ relative to the frame $\mathcal{T}, \hat{\mathbf{q}}_{c t}\left(t_{k}\right)$, can be calculated at any time. Accordingly, the estimated quaternion of the frame $\mathcal{T}$ relative to the frame $\mathcal{C}$ is

$$
\hat{\mathbf{q}}_{t c}\left(t_{k}\right)=\left[\begin{array}{ll}
-\hat{\varrho}_{c t}^{T}\left(t_{k}\right) & \hat{q}_{c t 4}\left(t_{k}\right)
\end{array}\right]^{T}
$$

\section{Position and velocity of the CM estimation of the target}

Assume the $\mathrm{CM}$ of the target moves approximately at a constant velocity in a short sampling interval $\Delta t$, such that

$$
\left\{\begin{array}{l}
\boldsymbol{\rho}_{0}\left(t_{k}\right)=\boldsymbol{\rho}_{0}\left(t_{k}-\Delta t\right)+\dot{\boldsymbol{\rho}}_{0}\left(t_{k}-\Delta t\right) \cdot \Delta t \\
\dot{\boldsymbol{\rho}}_{0}\left(t_{k}\right)=\dot{\boldsymbol{\rho}}_{0}\left(t_{k}-\Delta t\right)
\end{array}\right.
$$

Define state as $\mathbf{X}=\left[\begin{array}{lll}\mathbf{r}_{i}^{T} & \boldsymbol{\rho}_{0}^{T} & \dot{\boldsymbol{\rho}}_{0}^{T}\end{array}\right]^{T}$, which satisfies

$$
\mathbf{X}\left(t_{k}-j \cdot \Delta t\right)=\mathbf{A}^{-j} \mathbf{X}\left(t_{k}\right), k-c \leq j<k
$$

where $j$ is a positive integer, $c$ is a time period, and

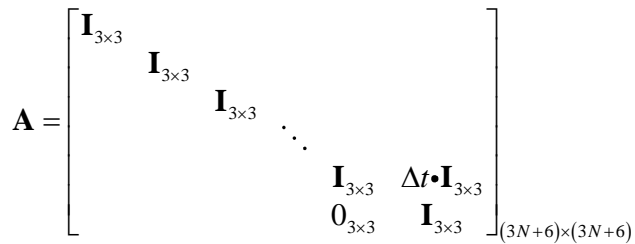

The measurement model can be written as

$$
\mathbf{Y}\left(t_{k}-j \cdot \Delta t\right)=\mathbf{C}\left(t_{k}-j \cdot \Delta t\right) \mathbf{A}^{-j} \mathbf{X}\left(t_{k}\right), k-c \leq j<k
$$

with

$$
\begin{gathered}
\mathbf{Y}\left(t_{k}\right)=\left[\begin{array}{lcc}
\mathbf{Y}_{1}\left(t_{k}\right)^{T} & \ldots & \mathbf{Y}_{i}\left(t_{k}\right)^{T}
\end{array}\right]^{T}, 1 \leq i \leq N \\
\mathbf{C}\left(t_{k}\right)=\left[\begin{array}{ccc}
\hat{\mathbf{R}}_{t}^{c}\left(t_{k}\right) \\
\vdots & \hat{\mathbf{R}}_{t}^{c}\left(t_{k}\right) & I_{3 \times 3} \\
& I_{3 \times 3} \\
{\left[\hat{\boldsymbol{\omega}}_{t c}^{\mathrm{c}}\left(t_{k}\right) \times\right]} & \hat{\mathbf{R}}_{t}^{c}\left(t_{k}\right) & I_{3 \times 3} \\
\vdots & & I_{3 \times 3} \\
& {\left[\hat{\boldsymbol{\omega}}_{t c}^{\mathrm{c}}\left(t_{k}\right) \times\right] \hat{\mathbf{R}}_{t}^{c}\left(t_{k}\right)} & I_{3 \times 3}
\end{array}\right]
\end{gathered}
$$

The state $\mathbf{X}$ can be estimated by LS given as

$$
\hat{\mathbf{X}}\left(t_{k}\right)=\left(\mathbf{H H}^{T}\right)^{-1} \mathbf{H}^{T} \mathbf{Y}
$$

where

$$
\mathbf{H}=\left[\begin{array}{c}
\mathbf{C}\left(t_{k}\right) \\
\mathbf{C}\left(t_{k}-\Delta t\right) \mathbf{A}^{-1} \\
\vdots \\
\mathbf{C}\left(t_{k}-j \cdot \Delta t\right) \mathbf{A}^{-1} \\
\vdots \\
\mathbf{C}\left(t_{k}-c \cdot \Delta t\right) \mathbf{A}^{-1}
\end{array}\right], \mathbf{Y}=\left[\begin{array}{c}
\mathbf{Y}\left(t_{k}\right) \\
\mathbf{Y}\left(t_{k}-\Delta t\right) \\
\vdots \\
\mathbf{Y}\left(t_{k}-j \cdot \Delta t\right) \\
\vdots \\
\mathbf{Y}\left(t_{k}-c \cdot \Delta t\right)
\end{array}\right]
$$

\section{Inertia ratio estimation}

In the absence of external torques, the angular momentum of the target is constant in the inertial frame. Since the frame $\mathcal{C}$ is assumed to be aligned with the inertial frame in this paper, the angular momentum of the target is constant in the frame $\mathcal{C}$, which is denoted as $\mathbf{h}^{c}$. At time instant $t$,

$$
\mathbf{R}_{c}^{t}(t) \mathbf{h}^{c}(t)=\mathbf{I} \omega_{t c}^{t}(t)
$$

where $\boldsymbol{\omega}_{t c}^{t}(t)=\mathbf{R}_{c}^{t}(t) \boldsymbol{\omega}_{t c}^{c}(t)$

Define the inertia vector as

$$
\mathbf{x}=\left[\begin{array}{llll}
\mathbf{I}^{* T} & h_{x}^{c} & h_{y}^{c} & h_{y}^{c}
\end{array}\right]^{T}
$$

with $\mathbf{I}^{*}=\left[\begin{array}{llllll}I_{x x} & I_{x y} & I_{x z} & I_{y y} & I_{y z} & I_{z z}\end{array}\right]^{T},(21)$ can be rewritten as

$$
\mathbf{A x}=\mathbf{0}
$$

where

$$
\mathbf{A}=\left(\begin{array}{c|c}
\Omega^{t}\left(t_{1}\right) & \mathbf{R}_{c}^{t}\left(t_{1}\right) \\
\vdots & \vdots \\
\Omega^{t}\left(t_{M}\right) & \mathbf{R}_{c}^{t}\left(t_{M}\right)
\end{array}\right)
$$


with

$$
\Omega^{b}(t)=\left(\begin{array}{cccccc}
\boldsymbol{\omega}_{t c x}^{t}(t) & \boldsymbol{\omega}_{t c y}^{t}(t) & \boldsymbol{\omega}_{t c z}^{t}(t) & 0 & 0 & 0 \\
0 & \boldsymbol{\omega}_{t c x}^{t}(t) & 0 & \boldsymbol{\omega}_{t c y}^{t}(t) & \boldsymbol{\omega}_{t c}^{t}(t) & 0 \\
0 & 0 & \boldsymbol{\omega}_{t c x}^{t}(t) & 0 & \boldsymbol{\omega}_{t c y}^{t}(t) & \boldsymbol{\omega}_{t c z}^{t}(t)
\end{array}\right)
$$

Equation (23) can be solved in the least square, which means that the solution should minimize

$$
f(\mathbf{x})=\|\mathbf{A} \mathbf{x}\|_{2}^{2}=(\mathbf{A} \mathbf{x})^{T} \mathbf{A} \mathbf{x}=\mathbf{x}^{T} \mathbf{A}^{T} \mathbf{A} \mathbf{x}
$$

Obviously $f(\mathbf{x})$ has the minimum when

$$
\mathbf{B x}=\mathbf{0}
$$

with $\mathbf{B}=\mathbf{A}^{T} \mathbf{A} \in \mathbb{R}^{9 \times 9}$.

Reordering such that

$$
\mathbf{B}=\left[\begin{array}{ll}
b_{11} & \mathbf{b}_{1}^{T} \\
\mathbf{b}_{1} & \mathbf{B}_{r}
\end{array}\right]
$$

where $b_{11} \in \mathbb{R}^{+}, \mathbf{b}_{1} \in \mathbb{R}^{8 \times 1}$, and $\mathbf{B}_{r} \in \mathbb{R}^{8 \times 8}$. Setting the first variable in $\mathbf{x}$ to 1 , i.e. $\mathbf{x}=\left[\begin{array}{ll}1 & \mathbf{x}_{r}^{T}\end{array}\right]^{T},(26)$ is reduced to

$$
\mathbf{B}_{r} \mathbf{x}_{r}=-\mathbf{b}_{1}
$$

However, there are physical constraints between the elements of the inertia tensor $\mathbf{I}$. Assuming that $I_{x x}$ is the largest diagonal element of $\mathbf{I}$, the following constraints hold,

$$
\left\{\begin{array}{l}
0<I_{y y}<I_{x x} \\
0<I_{z z}<I_{x x} \\
I_{y y}+I_{z z}>I_{x x} \\
2\left|I_{x y}+I_{x z}+I_{y z}\right|<I_{x x}+I_{y y}+I_{z z}
\end{array}\right.
$$

Since $\mathbf{B}_{r}$ is also a positive-definite matrix. Therefore, the problem is a convex quadratic programming problem with convex quadratic function $q\left(\mathbf{x}_{r}\right)=\frac{1}{2} \mathbf{x}_{r}^{T} \mathbf{B}_{\mathbf{r}} \mathbf{x}_{r}+\mathbf{b}_{1}^{T} \mathbf{x}_{r} \quad$ and inequality constraints (29) by setting the first variable in $\mathbf{x}$ to 1 . The interior-point-convex method is adopted here to solve this convex quadratic programming problem.

\section{Numerical Simulations}

In this section, numerical simulations are performed to verify the proposed estimation algorithm. The estimation errors are defined as:

$$
\begin{gathered}
e_{\rho_{0}}=\frac{\left\|\hat{\boldsymbol{\rho}}_{0}-\boldsymbol{\rho}_{0}\right\|_{2}}{\left\|\boldsymbol{\rho}_{0}\right\|_{2}}, e_{\dot{\rho}_{0}}=\frac{\left\|\hat{\boldsymbol{\rho}}_{0}-\dot{\boldsymbol{\rho}}_{0}\right\|_{2}}{\left\|\dot{\boldsymbol{\rho}}_{0}\right\|_{2}}, e_{\boldsymbol{\omega}_{t c}^{c}}=\frac{\left\|\hat{\boldsymbol{\omega}}_{t c}^{c}-\boldsymbol{\omega}_{t c}^{c}\right\|_{2}}{\left\|\boldsymbol{\omega}_{t c}^{c}\right\|_{2}} \\
e_{\mathbf{r}_{i}}=\frac{\left\|\hat{\mathbf{r}}_{i}-\mathbf{r}_{i}\right\|_{2}}{D}, e_{\mathbf{I}_{i}}=\frac{\left\|\hat{\mathbf{I}}_{i}^{*}-\mathbf{I}_{i}^{*}\right\|}{\left\|\mathbf{I}_{i}^{*}\right\|}
\end{gathered}
$$

where the superscripts " $\bullet$ " and "." denote the estimated value and true value, respectively, \|\|$_{2}$ is the norm of a vector, $\mathbf{I}_{i}^{*}$ is the component of the normalization of $\mathbf{I}^{*}$, and $D$ is a reference dimension. The relative attitude estimation error is defined as

$$
e_{\theta}=2 \cos ^{-1}\left(q_{e 4}\right)
$$

\begin{tabular}{|c|c|c|}
\hline \multicolumn{2}{|c|}{ Simulation parameter } & Values \\
\hline \multirow{4}{*}{$\begin{array}{c}\text { Camera } \\
\text { parameters }\end{array}$} & Baseline length & $1 \mathrm{~m}$ \\
\hline & Focal length & $0.1 \mathrm{~m}$ \\
\hline & Image noise & 0.2 pixel \\
\hline & Pixel Size & $2.55 \mu \mathrm{m}$ \\
\hline \multirow{4}{*}{$\begin{array}{c}\text { Initial } \\
\text { state }\end{array}$} & $\begin{array}{l}\text { Initial relative angular } \\
\text { velocity }\end{array}$ & {$\left[\begin{array}{llll}1.5 & 2.3 & -1.9\end{array}\right] \mathrm{rad} / \mathrm{s}$} \\
\hline & Initial relative attitude & {$\left[\begin{array}{lllll}0 & 0 & 0 & 1\end{array}\right]^{\mathrm{T}}$} \\
\hline & Initial position & {$\left[\begin{array}{llll}20 & 60 & 30\end{array}\right] \mathrm{m}$} \\
\hline & Initial velocity & {$\left[\begin{array}{lll}-0.01 & -0.01 & -0.02] \mathrm{m} / \mathrm{s}\end{array}\right.$} \\
\hline
\end{tabular}

where $q_{e 4}$ is the scalar part of the error quaternion defined by $\mathbf{q}_{e}=\mathbf{q}_{c c} \otimes \hat{\mathbf{q}}_{t c}$.

In the simulation, the sample time step is assumed to be $1 \mathrm{~s}$, the total simulation period is $3,000 \mathrm{~s}$, and the time period is 10 , the number of feature points is 4 and the reference dimension is $3 \mathrm{~m}$, the other conditions are listed in Table I.

Table I Simulation parameters.
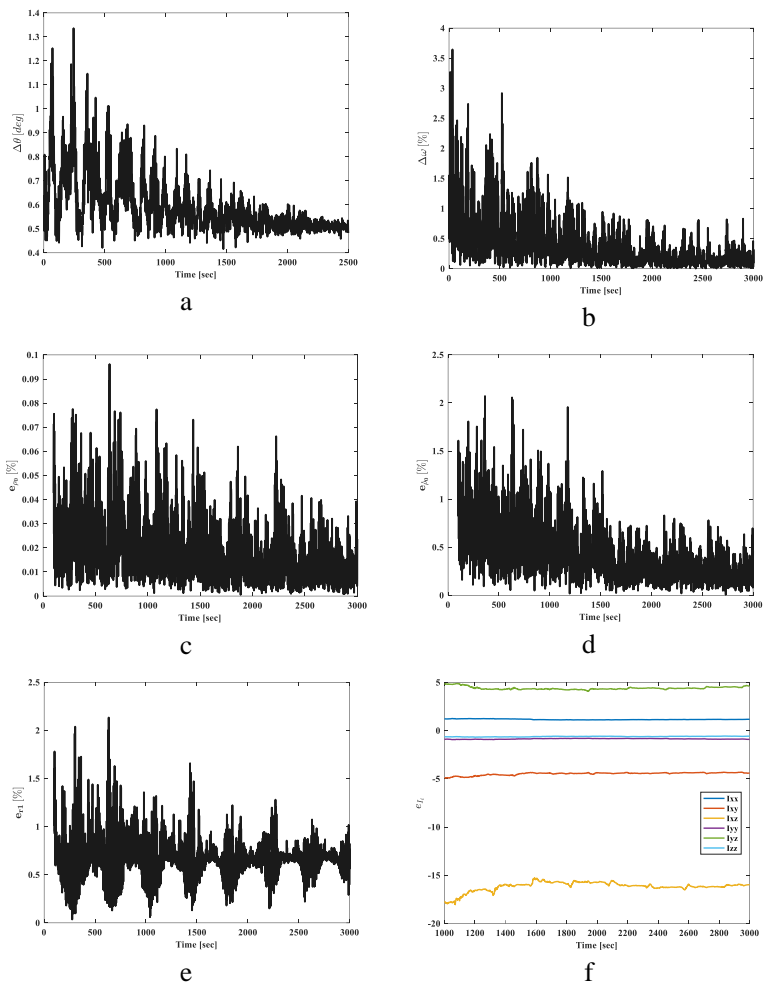

Fig. 2. Error of estimation. a) error of relative attitude, b) error of relative angular velocity, c) error of position for $\mathrm{CM}$ of the target, d) error of velocity for $\mathrm{CM}$ of the target, e) error of position estimation of one feature point, f) error of inertia ratio estimation.

As shown in Fig. 2, the relative state and inertia ratio estimation can get high accuracy by the designed algorithm. The relative attitude error is less than $2.5 \%$, the relative angular velocity error is no more than $4 \%$, and the position and velocity errors of the $\mathrm{CM}$ are less than $0.1 \%$ and $2.5 \%$, respectively, and the position error of one feature relative the reference dimension is less than $2.5 \%$, the error of the normalized moments of inertia is of magnitude $18 \%$. In fact, the number of the attitudes and angular velocities influence the accuracy of inertia ratio which is based on a least square optimization. 


\section{CONCLUSIONS}

This paper develops an effective algorithm to estimate the relative pose, motion, $\mathrm{CM}$ and inertia ratio of an unknown target based on the consecutive images obtained from stereoscopic vision. The positions and velocities of the detected feature points on the target are estimated first, and then the estimation of the pose, motion, CM and inertia ratio. The advantage of this algorithm is its ability to estimate the CM, inertia ratio and the constellation of feature points of the target simultaneously without prior knowledge of the target. The simulation results show that the proposed algorithm can get a good estimation accuracy in acceptable measurement noise level.

\section{ACKNOWLEDGMENT}

The work of this paper is by National Natural Science Foundation of China under grant 61503304, grant 61790552, grant 61703343, and the Discovery Grant of the Natural Sciences and Engineering Research Council of Canada (NSERC).

\section{REFERENCES}

[1] Barnhart, David, et al. , "XSS-10 micro-satellite demonstration," AIAA Defense and Civil Space Programs Conference and Exhibit 2013.

[2] Nishida, S. I., Kawamoto, S., Okawa, Y., Terui, F., and Kitamura, S. "Space debris removal system using a small satellite," Acta Astronaut., Vol. 65, No. 1-2, 2009.

[3] Zhang, L., Zhang, S., Yang, H., Cai, H., and Qian, S. "Relative attitude and position estimation for a tumbling spacecraft," Aerospace Science and Technology Vol. 42, pp. 97-105, 2015.

[4] Ma, C., Dai, H., and Yuan, J. "Estimation of inertial characteristics of tumbling spacecraft using constant state filter," Advances in Space Research, 2017, in press.

[5] Segal, S., Carmi, A., and Gurfil, P. "Stereovision-Based Estimation of Relative Dynamics Between Noncooperative Satellites: Theory and
Experiments," Control Systems Technology, IEEE Transactions on Vol. 22, No. 2, pp. 568-584, 2014.

[6] Xu, W., Liang, B., Li, C., and Xu, Y. "Autonomous rendezvous and robotic capturing of non-cooperative target in space," Robotica, Vol. 28, No. 5, 2010.

[7] Terui, F., Kamimura, H., and Nishida, S. "Motion Estimation to a Failed Satellite on Orbit using Stereo Vision and 3D Model Matching," Control, Automation, Robotics and Vision, 2006. ICARCV '06. 9th International Conference on. 2006, pp. 1-8.

[8] Dehann, F., Brent, T., Steve, U., and Alvar Saenz, O. "Vision-Based Relative Navigation and Control for Autonomous Spacecraft Inspection of an Unknown Object," AIAA Guidance, Navigation, and Control (GNC) Conference. American Institute of Aeronautics and Astronautics, 2013.

[9] Du, X., Liang, B., Xu, W., \& Qiu, Y., "Pose measurement of large noncooperative satellite based on collaborative cameras,". Acta Astronautica, 68(11-12), 2047-2065, 2011.

[10] Dong, Gangqi, and Z. H. Zhu. "Autonomous robotic capture of noncooperative target by adaptive extended Kalman filter based visual servo," Acta Astronautica 122:209-218, 2016.

[11] Dong, Gangqi, and Z. H. Zhu. "Incremental inverse kinematics based vision servo for autonomous robotic capture of non-cooperative space debris," Advances in Space Research 57.7:1508-1514, 2016.

[12] Lichter, M. D., and S. Dubowsky. "State, shape, and parameter estimation of space objects from range images," IEEE International Conference on Robotics and Automation, 2004. Proceedings. ICRA IEEE, 2004: 2974-2979 Vol.3.

[13] Tweddle, B. E. "Computer Vision-Based Localization and Mapping of an Unknown, Uncooperative and Spinning Target for Spacecraft Proximity Operations," Ph.D. dissertation, Massachusetts Institute of Technology, 2013.

[14] Shay, S., and Pini, G. "Stereoscopic Vision-Based Spacecraft Relative State Estimation," AIAA Guidance, Navigation, and Control Conference. American Institute of Aeronautics and Astronautics, 2009.

[15] Ma, Ou, H. Dang, and K. Pham. "On-Orbit Identification of Inertia Properties of Spacecraft Using a Robotic Arm," Journal of Guidance Control \& Dynamics 31.6:1761-1771, 2008.

[16] Kim, Dong Hoon, D. G. Choi, and H. S. Oh. "Inertia Estimation of Spacecraft Based on Modified Law of Conservation of Angular Momentum," Journal of Astronomy \& Space Sciences 27.1: 353-357, 2010 . 